JOURNAL OF SYNCHROTRON RADIATION

ISSN 1600-5775

Received 17 March 2021

Accepted 11 January 2022

Edited by A. Momose, Tohoku University, Japan

Keywords: cryo-cooled Si monochromator; deformation modelling; lensing effect; cooling design.

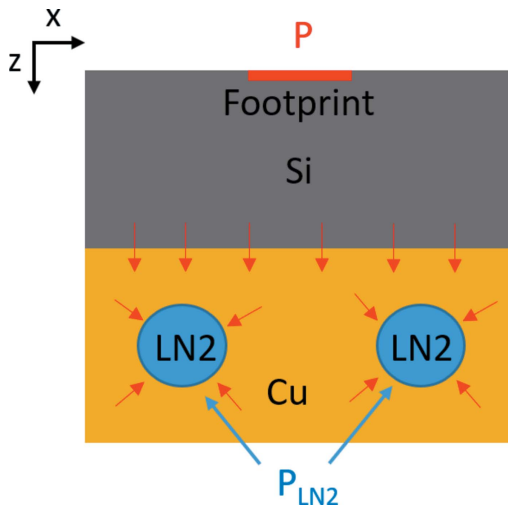

OPEN Ә ACCESS

\section{Cryo-cooled silicon crystal monochromators: a study of power load, temperature and deformation}

\author{
Hossein Khosroabadi,* Lucia Alianelli, Daniel G. Porter, Steve Collins and \\ Kawal Sawhney
}

Diamond Light Source, Diamond House Harwell Science and Innovation Campus, Oxfordshire OX11 ODE, United Kingdom. *Correspondence e-mail: hossein.khosroabadi@diamond.ac.uk

Crystal monochromators are often the primary optics in hard X-ray synchrotron beamlines. Management of power load is central to their design. Strict requirements on stability and deformation are to be met, as new-generation synchrotron sources deliver brighter beams of X-rays. This article sets out to illustrate an overall picture of the deformation caused by heat load in a cryocooled Si crystal monochromator using first principles. A theoretical model has been developed to predict the temperature distribution and surface deformation by applying intrinsic properties of $\mathrm{Si}$ material and the cooling system parameters. The model explains the universal behaviour of crystal slope error versus absorbed power; it has been benchmarked against experimental data and used to interpret finite-element analysis of cryogenically cooled crystals.

\section{Introduction}

Cryo-cooled silicon crystals have been used for decades as monochromators in hard X-ray synchrotron beamlines. Relatively large thermal conductivity at low temperature and zero linear thermal expansion coefficient at temperatures $T_{\text {zero }} \simeq 125 \mathrm{~K}$ result in low crystal deformation compared with other perfect crystal materials, or to water-cooled silicon (Chumakov et al., 2004; Zhang et al., 2003; Marot et al., 1992; Yates et al., 2010). Well designed cryo-cooling systems successfully minimize the surface deformation even under high power load from intense undulator sources. However, ongoing upgrades of synchrotron photon sources require further improvements of optical components. The aim is to conserve the natural collimation and brightness of synchrotron beams in lower-emittance machines (Huang et al., 2014; Brumund et al., 2021; Zhang et al., 2013).

Considerable increase of incident power $P$ and power density $P_{\mathrm{d}}$ on monochromators is currently observed on some hard X-ray beamlines at Diamond Light Source (DLS), equipped with new higher-magnetic-field undulators. Power will increase further with the new Diamond-II machine, when the ring energy is increased from 3 to $3.5 \mathrm{GeV}$ (Diamond-II Conceptual Design Report: https://www.diamond.ac.uk/ Home/About/Vision/Diamond-II.html). Effective control of power is fundamental and can be accomplished by use of smaller primary slits and filter materials. An optimized crystal cooling design will reduce optics deformations, prevent changes in diffracted beam wavefront and improve focusing.

Finite-element analysis (FEA) has been widely used to investigate thermal deformation of beamline optics such as mirrors and monochromators (Zhang et al., 2003, 2013; Yates et al., 2010; Dolbnya et al., 2019; Cheng et al., 2015). It can be a 
time-consuming analysis, as the predicted crystal deformation varies considerably with changing scenarios, such as incident power and cooling parameters. FEA is a powerful tool in the design of cooling systems, with the caveat that simulations may need interpretation depending on the assumptions and parameters used. For instance, in the indirect cooling geometry, contact conductance between crystal and copper cooling block is not easily determined, and FEA provides a wide range of slope errors depending on input values. In situ surface deformation measurements (Kazimirov et al., 2007, 2008; Revesz et al., 2007; Rutishauser et al., 2013) require specialized set-ups, which are not readily available or feasible for any monochromator system.

A simplified model of temperature spatial distribution and deformation is presented in the first part of the article. It explains the properties of cryo-cooled Si crystals which are observed experimentally and predicted by FEA; therefore, it can be used for an initial assessment of cooling requirements. An experimental study of the I16 beamline monochromator (Collins et al., 2010) at DLS is described in the second part of the article. The model reproduces the experimental data, therefore showing that, in combination with FEA, it is a simple and intuitive tool to design cooling of advanced synchrotron optics.

\section{Theoretical study}

\subsection{Modelling}

Temperature and crystal deformation as a function of time, power and power density are presented in this section. Solutions for the principal quantities such as $T_{\mathrm{b}}$, the crystal body temperature, $T_{\mathrm{p}}$, the peak surface temperature, $T_{\mathrm{a}}$, the temperature at the beam footprint margin, and $\Delta L$, the thermal strain are derived in Appendix $A$.

The model, schematically illustrated in Fig. 1, is valid for an indirectly cryo-cooled Si crystal but can be extended to the direct cooling geometry. Whilst $T_{\mathrm{b}}$ stays nearly constant, the temperature at the beam footprint area evolves rapidly (Zhang et al., 2013) when this area is much smaller than the crystal surface.

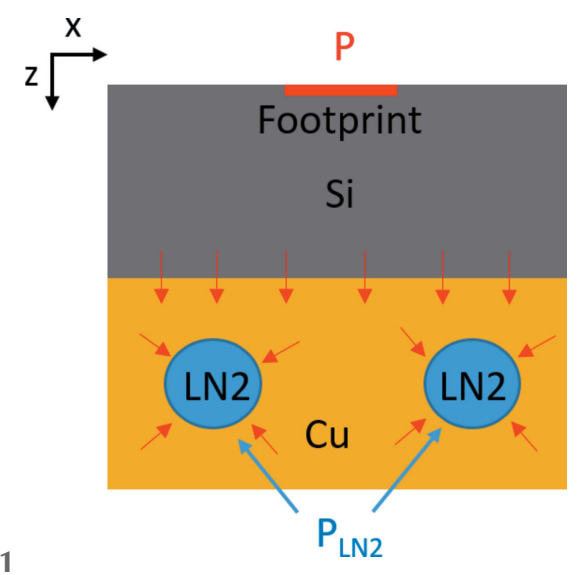

Figure 1

Schematic view of the indirect cryo-cooled monochromator. The red arrows show heat flow from the crystal to the cooling LN2 channels.
The following coupled differential equations describe the temporal evolution of the average temperature of the cooling copper block $T_{\mathrm{Cu}}$ and Si crystal $T$,

$$
\begin{aligned}
m_{\mathrm{Si}} C_{\mathrm{Si}} \frac{\partial T}{\partial t}= & P-k_{\mathrm{SiCu}} A_{\mathrm{SiCu}}\left[T(t)-T_{\mathrm{Cu}}(t)\right], \\
m_{\mathrm{Cu}} C_{\mathrm{Cu}} \frac{\partial T_{\mathrm{Cu}}}{\partial t}= & k_{\mathrm{SiCu}} A_{\mathrm{SiCu}}\left[T(t)-T_{\mathrm{Cu}}(t)\right] \\
& -k_{\mathrm{CuLN} 2} A_{\mathrm{CuLN} 2}\left[T_{\mathrm{Cu}}(t)-T_{\mathrm{LN} 2}(t)\right],
\end{aligned}
$$

where $m_{\mathrm{Si}(\mathrm{Cu})}$ and $C_{\mathrm{Si}(\mathrm{Cu})}$ are the mass and specific heat capacity of $\mathrm{Si}(\mathrm{Cu}) ; k_{\mathrm{SiCu}}$ and $A_{\mathrm{SiCu}}$ are the conductance and area of the $\mathrm{Si}-\mathrm{Cu}$ contact; similar symbols denote $\mathrm{Cu}$ and liquid nitrogen (LN2). The analytical solution for $T(t)$ at constant incident power and LN2 temperature is

$$
T(t)=T_{\mathrm{b}}+T_{1} \exp \left(-t / \tau_{+}\right)+T_{2} \exp \left(-t / \tau_{-}\right)
$$

Expressions for $T_{\mathrm{b}}, \tau_{+}$and $\tau_{-}$are derived in Appendix $A$. Crystal temperature increases exponentially with $\tau_{+}$and $\tau_{-}$ time scales, until it reaches an asymptotic value $T_{\mathrm{b}}$. For a constant $T_{\mathrm{Cu}}$, only the first exponential term remains in equation (3).

Heat due to absorbed power is transferred with radial symmetry in the crystal, in good approximation. Constant temperature layers at a distance $r$ from the centre of the beam footprint can be defined (Zhang et al., 2003; Yates et al., 2010). The relationship between radial power and crystal temperature gradient $\partial T / \partial r$ can therefore be expressed as

$$
P_{\text {radial }}(r)=-k_{\mathrm{Si}}[T(r)] A(r) \frac{\partial T}{\partial r} .
$$

Here, $k_{\mathrm{Si}}$ and $A$ are the Si thermal conductivity and area of the layer located at distance $r$. $T(r)$ can be obtained by analytical integration for the circular footprint case, or numerically for the elliptical case. Large changes in $A$ and $k_{\mathrm{Si}}$ with $r$ lead to fast convergence of $T(r)$ to $T_{\mathrm{b}}$ at distances $r \simeq 4 a$, where $a$ is the radius of the beam footprint. A numerical solution can be determined for any power distribution. The solutions for uniform power density distribution, a good approximation for undulator beams at third-generation sources, are derived in the Appendix $A$.

Crystal surface deformation, for the stretching and bending components, is calculated via the following integrals,

$$
\begin{aligned}
\theta_{\text {stretch }} & =\frac{\int_{0}^{d} \alpha(T)[T(x=0, z)-T(x=a, z)] \mathrm{d} z}{a}, \\
\theta_{\text {bend }} & =\frac{\int_{0}^{l} \alpha(T)[T(x, z=0)-T(x, z=d)] \mathrm{d} x}{d},
\end{aligned}
$$

where $\alpha(T)$ denotes the Si linear expansion coefficient, $d \simeq 2 a$ is the integration depth and $l$ is the length over which the crystal temperature is varying considerably.

Stretching is the result of the crystal freely expanding perpendicularly to the beam footprint. Since the much larger crystal bulk, at temperature $T_{\mathrm{b}}$, does not expand freely along the surface, the region near the footprint is subject to strong bending. This is often the dominant contribution to defor- 
mation, as temperature gradients are higher along depth. Approximate values for the slope error $\theta$ are derived by assuming constant temperature at the footprint and within a crystal depth $d \simeq 2 a$. The bending term can be calculated via the thermal strain,

$$
\theta=\frac{\Delta L_{\mathrm{p}, \mathrm{b}}}{d}
$$

where

$$
\Delta L_{\mathrm{p}, \mathrm{b}}=\int_{T_{\mathrm{b}}}^{T_{\mathrm{p}}} l(T) \alpha(T) \mathrm{d} T
$$

\subsection{Discussion and applications}

The model is valid under the following assumptions: the beam footprint is smaller than the size of the crystal; power distribution has circular or elliptical symmetry; mechanical stress or other forces can be ignored; and the crystal is free to expand perpendicular to the surface. Temperature $T_{\mathrm{b}}$ should be determined either experimentally or from equation (9).

Use of the model has allowed: verification and interpretation of measurements and FEA data; estimation of slope errors for a wide range of incident beam power, power density and footprints (or Bragg angles); assessment of existing crystal cooling designs; preliminarily design of new cooling systems. Determination of the LN2 flow rate and precise geometry of the cooling channels are outside of the scope of this study. As the model has so far provided accuracy of order $10 \%$ to $20 \%$, compared with FEA or experimental data at DLS, the final monochromator design should rely on FEA.

Comparison with the published FEA temperature data from a crystal monochromator at the ESRF (Zhang et al., $2013)$ is presented in Fig. 2. We have used equations (14a) and (14b), with $T_{\mathrm{b}}$ extrapolated from the published data. Good agreement is observed, despite the approximations used to derive these solutions.

Slope error values, as obtained from equations (6) and (7), are plotted next to the published data (Zhang et al., 2013)

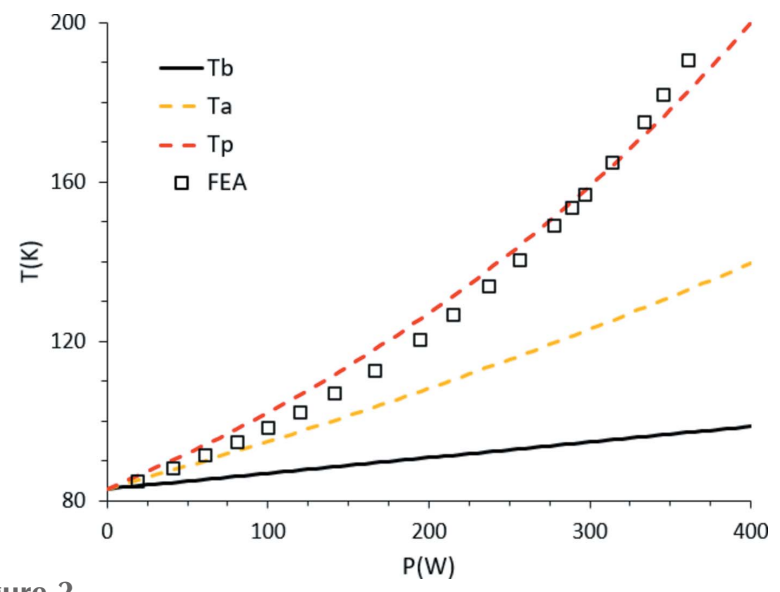

Figure 2

$T_{\mathrm{a}}, T_{\mathrm{p}}$ calculated from equations $(14 a)$ and $(14 b)$ and compared with FEA results (Zhang et al., 2013). We have used $a=1.7 \mathrm{~mm}$.

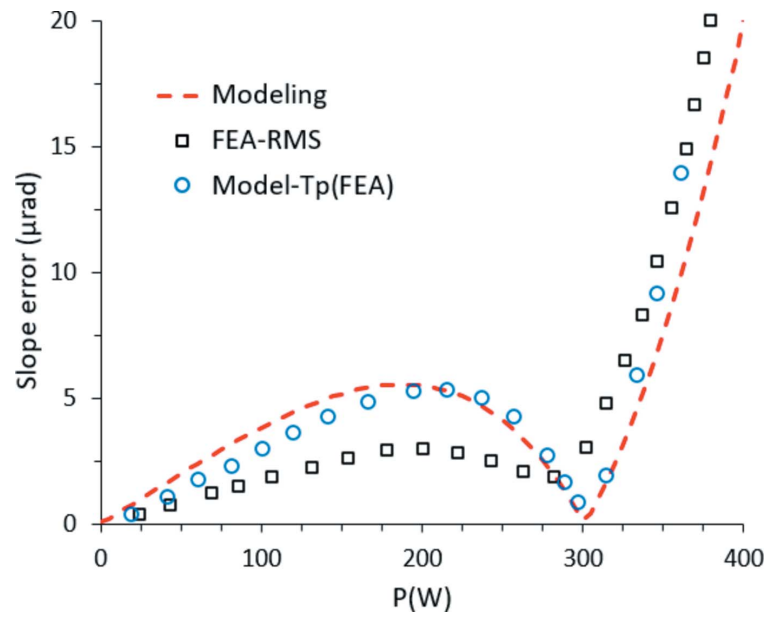

Figure 3

Slope error calculation from equations (6) and (7) (red dashes) compared with FEA results of Zhang et al. (2013). Blue circles are obtained from $T_{\mathrm{p}}$ values extrapolated from FEA.

in Fig. 3. Data trends are similar and deviation from FEA results are due to the complex dependence of the modelled phenomena on power distribution, total power, footprint, contact conductance and cooling design details.

The mechanism underlying the strongly non-linear crystal deformation is illustrated schematically in Fig. 4. The bending contribution causes three possible types of deformation: from concave shape at low power, to flat, and finally to highly convex at high power. This behaviour is caused by the unique

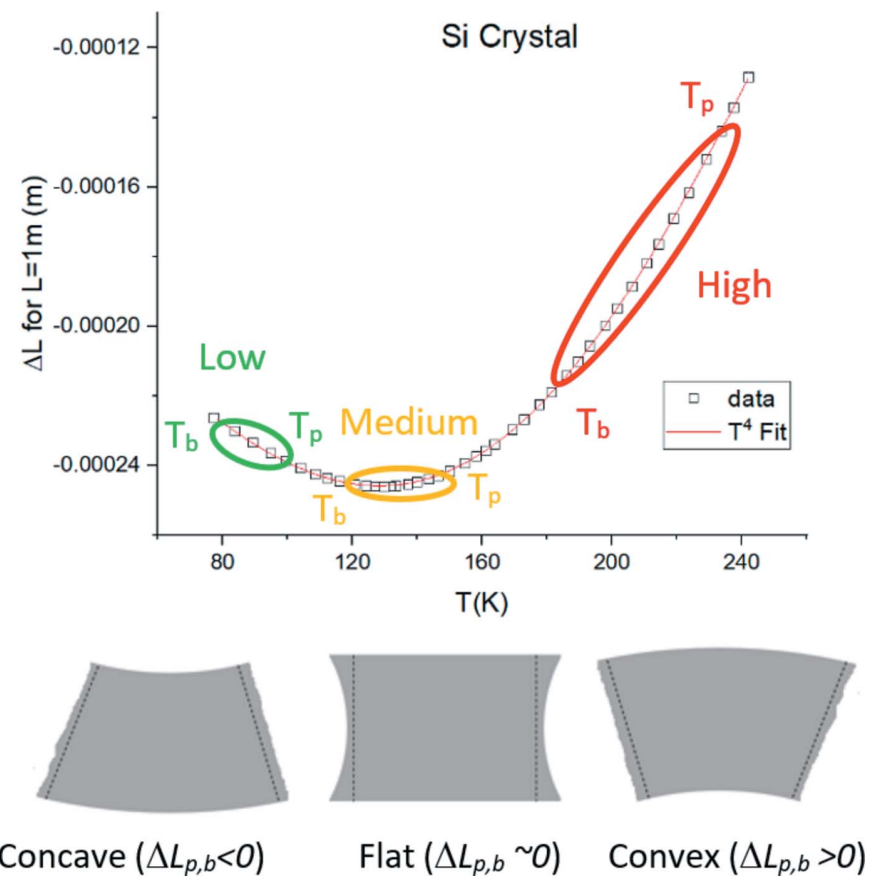

Figure 4

Top: length expansion of $\mathrm{Si}$ (squares) fitted by a $T^{4}$ polynomial (red line). The ellipses show the location of $T_{\mathrm{p}}$ and $T_{\mathrm{b}}$ on the graph for three different regions of low, medium and high power. Bottom: schematic shape of the crystal deformation from the concave to convex regime for the three power ranges. 

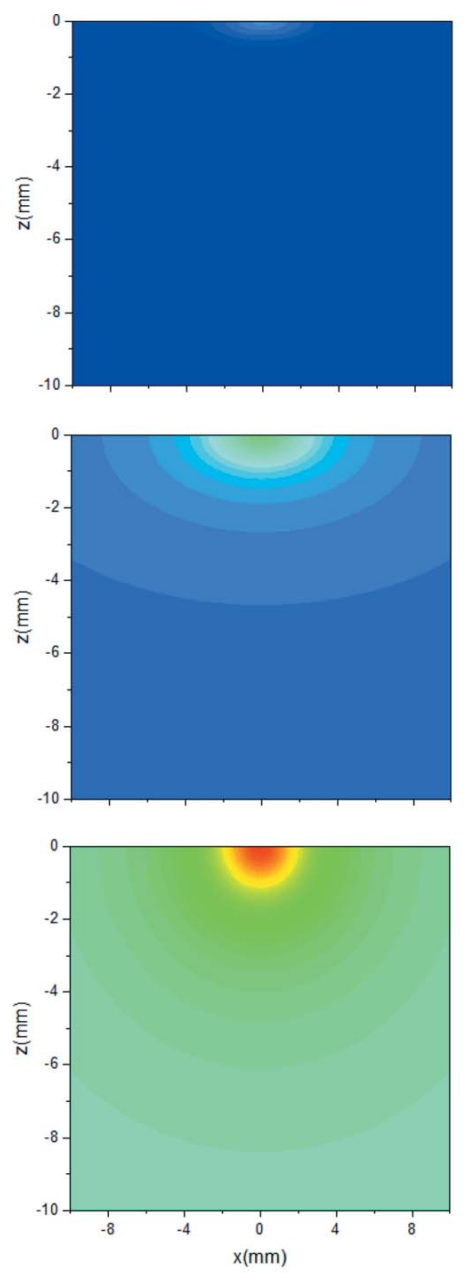

$\mathrm{T}(\mathrm{K})$

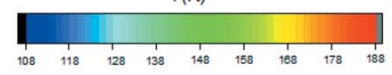

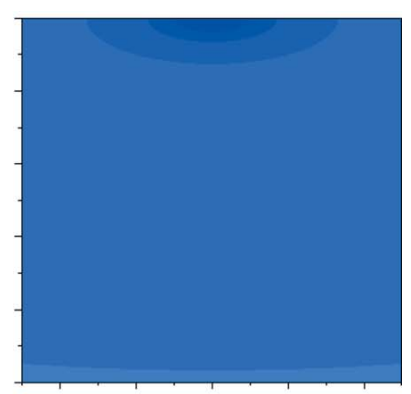
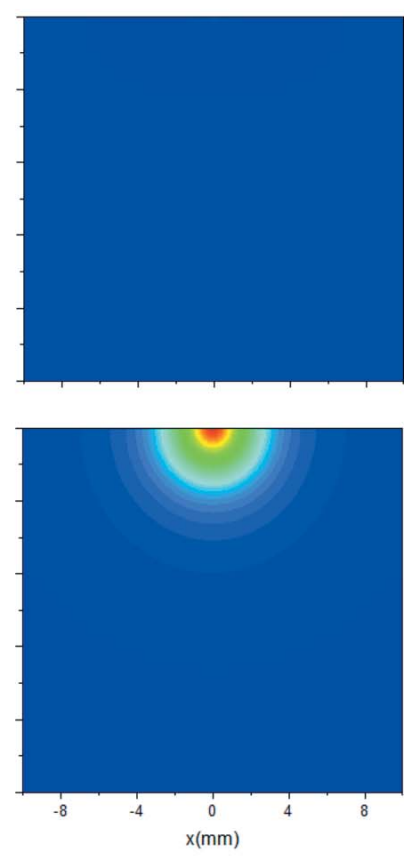

Thermal strain $\left(x 10^{-7} \mathrm{~m}\right)$

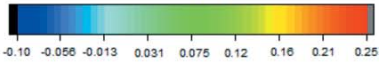

Figure 5

I16 Si crystal temperature and thermal strain calculated for different values of primary slit sizes, corresponding to $P=69 \mathrm{~W}, 116 \mathrm{~W}$ and $207 \mathrm{~W}$ (top to bottom) at fixed photon energy $E=3.5 \mathrm{keV}$. Left: temperature distribution. Right: thermal strain relative to $97 \mathrm{~K}$ ( or $P=0$ ).

properties of the $\mathrm{Si}$ thermal expansion coefficient and is universally observed in cryo-cooled Si crystals subject to heat load. The shape and amplitude of the deformation are determined by the characteristic temperatures $\left(T_{\mathrm{b}}\right.$ and $\left.T_{\mathrm{p}}\right)$ of a given crystal, in each scenario of power load and cooling. Efforts to keep the temperature at a beam footprint close to $T_{\text {zero }}$ do not result in minimum deformation. Deformation can be minimized, when temperatures $T_{\mathrm{b}}$ and $T_{\mathrm{p}}$ are symmetrical

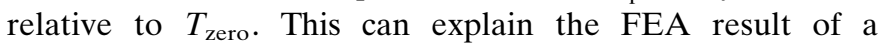
minimum surface deformation at $T_{\mathrm{p}} \simeq 150 \mathrm{~K}$ and $T_{\mathrm{b}} \simeq 95 \mathrm{~K}$ (Zhang et al., 2013).

Further accuracy is obtained solving equation (4) numerically. Details such as crystal shape and size can be ignored, under the usual assumption of the crystal being much larger than the beam footprint. Crystal stress can be calculated, if required, by including mechanical properties of Si such as bulk modulus tensor and Poisson ratio. Slope error amplitude is

calculated for $d=2 \min (a, b)$ and over the entire footprint area. Calculations have been cross-checked again with several FEA studies at DLS.

Numerical models of temperature and thermal strain for the I16 crystal monochromator at photon energy $E=3.5 \mathrm{keV}$ and varying incident power $(P=69 \mathrm{~W}, 116 \mathrm{~W}, 207 \mathrm{~W})$ are shown in Fig. 5. The respective calculated temperature values are: $T_{\mathrm{b}}=$ $108 \mathrm{~K}, 116 \mathrm{~K}$ and $132 \mathrm{~K} ; T_{\mathrm{p}}=121 \mathrm{~K}, 141 \mathrm{~K}$ and $188 \mathrm{~K}$. These examples illustrate the three different temperature scenarios, corresponding to the low, medium and high power: $\left(T_{\mathrm{b}}, T_{\mathrm{p}}\right)<$ $T_{\text {zero }} ; T_{\mathrm{b}}<T_{\text {zero }}<T_{\mathrm{p}} ;\left(T_{\mathrm{b}}, T_{\mathrm{p}}\right)>T_{\text {zero }}$.

The sign of the relative thermal strain can change along the crystal depth. Slope error amplitude is calculated by integrating the thermal strain. At low power, smaller strain occurs at the footprint than in the crystal bulk, resulting in concave bending. At medium power, the thermal expansion gradient is negligible and surface deformation is minimal. At high power, a large thermal expansion gradient causes a strong deformation with convex shape. Slope error increase with power is due to a stronger temperature gradient and thermal expansion coefficient. This explains the origin of the three deformation phases seen in previous studies (Zhang et al., 2003, 2013; Huang et al., 2014; Brumund et al., 2021). The power and temperature regime at which the crystal is nearly flat, also called the 'sweet spot', should be carefully determined to prevent transition to the next regime, where deformation increases steeply with power.

\section{Experimental study}

\subsection{Measurement details}

Undesirable changes of the focused beam size at the endstation are observed on beamline I16. The vertical beam size is strongly affected by power levels on the crystal monochromator, which are in turn dependent on undulator settings, primary slits size and monochromator Bragg angle.

The optical layout is shown in Fig. 6. A 2 m-long in-vacuum undulator (IVU) source produces $3.2 \mathrm{~kW}$ total power at its minimum gap $(5 \mathrm{~mm})$. The beamline utilizes a set of primary slits $\left(\mathrm{S}_{1}\right)$ at $23.6 \mathrm{~m}$ and a channel-cut $\mathrm{Si}(111)$ crystal monochromator at $25.5 \mathrm{~m}$ from the source. The crystal is cooled indirectly by flowing LN2 through two cooling channels inside the $\mathrm{Cu}$ block (Fig. 1). No filters or windows are used since the photon energy range extends to $E=2.5 \mathrm{keV}$. A set of vertical and horizontal focusing mirrors at 29.5 and $31 \mathrm{~m}$, respectively, focus the beam at the sample position at $50 \mathrm{~m}$ from the source.

Beam size and photon flux at the sample position were measured for different values of incident power. The crystal temperature $T_{\mathrm{b}}$ was continuously recorded by a thermocouple attached to the crystal side. The synchrotron ring current was nearly constant, $I=300 \mathrm{~mA}( \pm 1 \%$, due to top-up), and the power was varied by varying the selected photon energy, the undulator gap values and the size of the primary slits $S_{1}$, up to $5 \mathrm{~mm} \times 3 \mathrm{~mm}$. This multi-parameter approach gave detailed insight into the crystal response to power, compared with previous studies where ring current was the main variable 


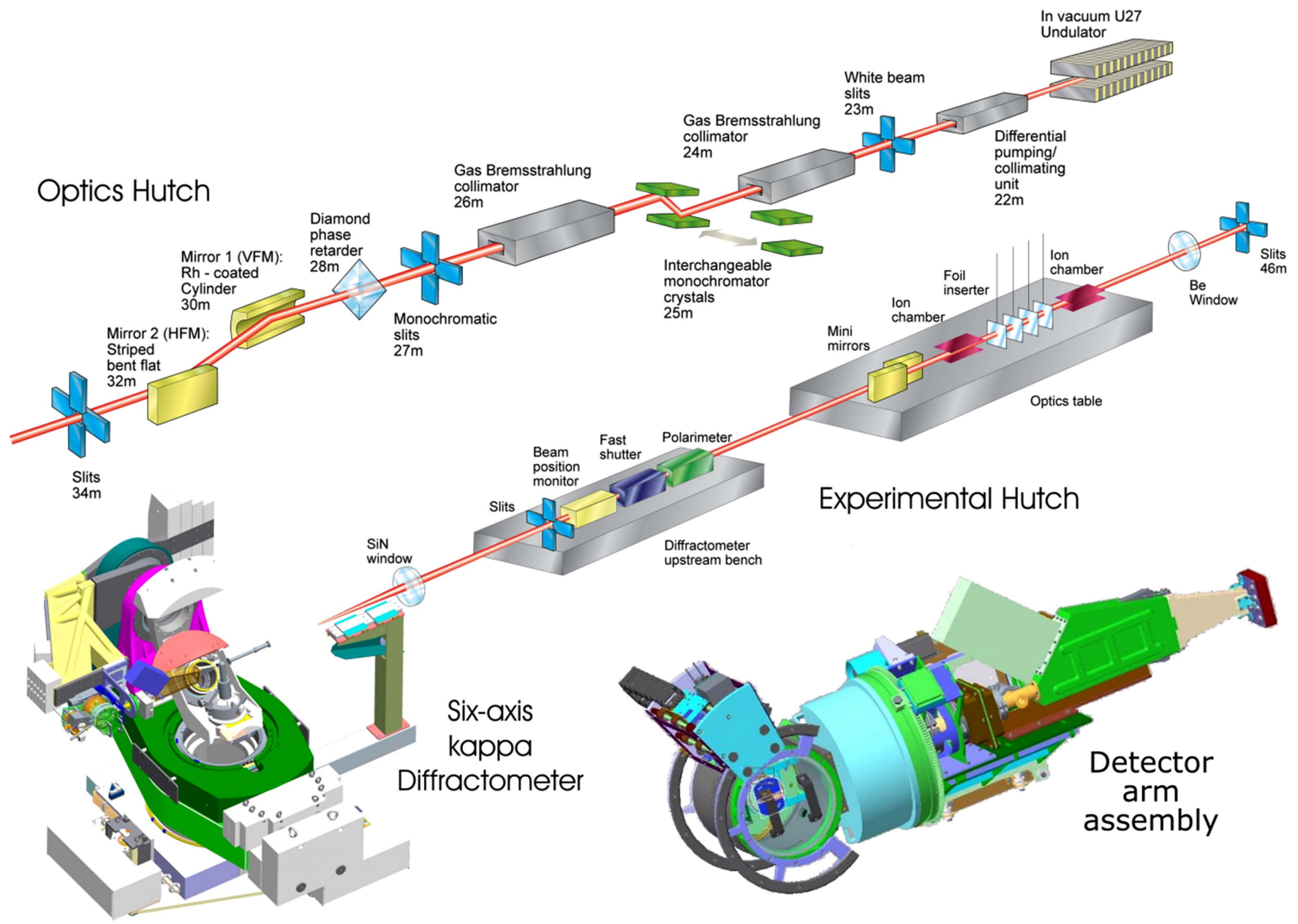

Figure 6

I16 beamline layout at DLS. The undulator radiation passes through primary adjustable slits and is nearly completely absorbed by the first Si crystal.

(Zhang et al., 2013; Antimonov et al., 2016). Power scenarios, calculated using the SPECTRA code (Tanaka \& Kitamura, 2001), are summarized in Table 1 . Absorbed power could be up to $10 \%$ lower than theoretical values (Zhang et al., 2013); however, this does not affect the following discussion.

\subsection{Results and discussion}

An example of the measured evolution of $T(t)$ is shown in Fig. 7: temperature increases exponentially from $96 \mathrm{~K}$ to $112 \mathrm{~K}$ with a time scale of about $100 \mathrm{~s}$. Data are well fitted by equation (3), even using the constant $T_{\mathrm{Cu}}(t)$ approximation. Fitting parameters at two gap values are summarized in Table 2 and we obtain $k_{\mathrm{SiCu}} \simeq 1820 \mathrm{~W} \mathrm{~m}^{-2} \mathrm{~K}^{-1}$ for this crystal, using equation (11).

Contact conductance $k_{\mathrm{SiCu}}$ is an important parameter, which is sometimes estimated experimentally. Several studies have previously suggested that an initial analytical estimation, followed by a comparison of the measured temperatures in the actual conditions with the theoretical prediction, can be successfully used to determine the contact conductance with the required accuracy (Yovanovich, 2005). To design cryo-
Table 1

Calculated average power density $P_{\mathrm{d}}$ at $\mathrm{S}_{1}$ and at the crystal surface for different values of photon energy and undulator deflection parameter $K_{\text {IVU }}$.

\begin{tabular}{lllll}
\hline$E(\mathrm{keV})$ & $K_{\mathrm{IVU}}$ & Harmonic & $\begin{array}{l}P_{\mathrm{d}} \text { at } \mathrm{S}_{1} \\
\left(\mathrm{~W} \mathrm{~mm}^{-2}\right)\end{array}$ & $\begin{array}{l}P_{\mathrm{d}} \text { on crystal } \\
\left.(\mathrm{W} \mathrm{mm})^{-2}\right)\end{array}$ \\
\hline 3.5 & 1.85 & 3 & 23.1 & 11.2 \\
5.0 & & & & \\
& 2.08 & 5 & 26.2 & 8.9 \\
& 1.34 & 3 & 15.8 & 5.4 \\
& & & 28.7 & 6.1 \\
& 2.27 & 9 & 23.3 & 5.0 \\
& 1.87 & 7 & 17.4 & 3.7 \\
& 1.39 & 5 & 6.1 & 1.3 \\
\hline
\end{tabular}

cooling systems, comparison of FEA with experiments should be used to reduce the uncertainty. Multiple measurements are necessary to determine an average value for $k_{\mathrm{SiCu}}$, as it often depends on $P$ and $P_{\mathrm{d}}$. Alternatively, it can be calculated from the slope of $T_{\mathrm{b}}$ versus $P$ as shown in Fig. 8. $T_{\mathrm{b}}$ increases approximately linearly at low to medium power $(P<150 \mathrm{~W})$ and at smaller rate at higher power. Such a slower increase 
Table 2

The fitted parameter for the single- and double-exponential models, using data in Fig. 7.

Other constant (or average) values used are: $C_{\mathrm{Si}}(105 \mathrm{~K}) \simeq 260 \mathrm{~J} \mathrm{~kg}^{-1} \mathrm{~K}^{-1}$, $m_{\mathrm{Si}}=0.21 \mathrm{~kg}, T_{\mathrm{LN} 2}=82 \mathrm{~K}, A_{\mathrm{SiCu}}=15 \mathrm{~cm}^{2}$.

\begin{tabular}{|c|c|c|c|c|c|c|c|c|c|}
\hline \multirow[b]{2}{*}{ Gap $(\mathrm{mm})$} & \multirow[b]{2}{*}{$P(\mathrm{~W})$} & \multicolumn{3}{|c|}{ One exponential term } & \multicolumn{5}{|c|}{ Two exponential terms } \\
\hline & & $T_{\mathrm{b}}$ & $T_{1}$ & $\tau_{+}$ & $T_{\mathrm{b}}$ & $T_{1}$ & $\tau_{+}$ & $T_{2}$ & $\tau_{-}$ \\
\hline 5.5 & 73 & 115 & -18 & 33 & 115 & -11 & 17 & -8 & 59 \\
\hline 7.0 & 60 & 111 & -14 & 32 & 112 & -10 & 17 & -6 & 59 \\
\hline
\end{tabular}

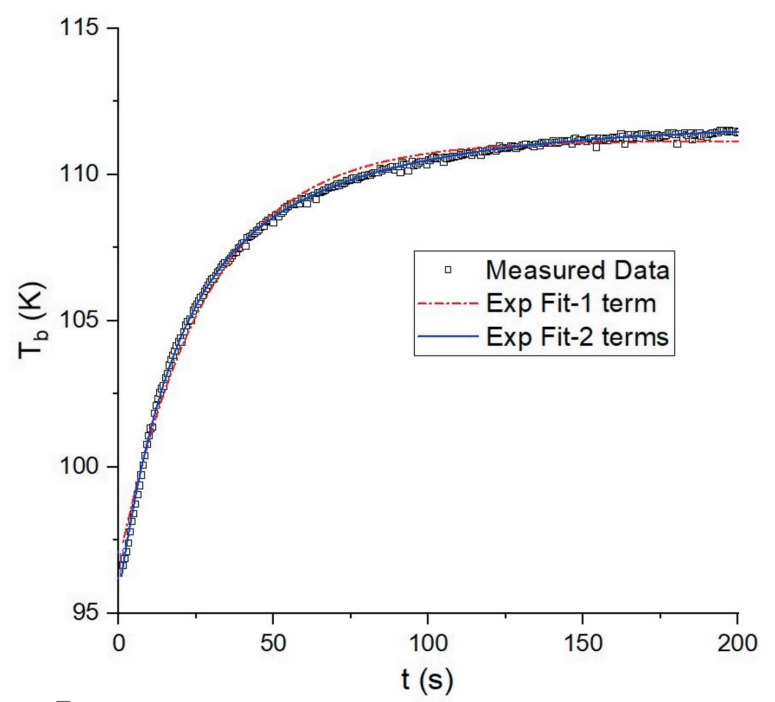

Figure 7

Temporal evolution of I16 crystal temperature for an undulator gap of $7.0 \mathrm{~mm}$ and $\mathrm{S}_{1}$ size of $2.5 \mathrm{~mm} \times 1.0 \mathrm{~mm}$. The red and blue lines are fit results with one and two exponential terms, respectively.

is probably due to higher cooling efficiency and increased effective $\mathrm{Cu}-\mathrm{Si}$ contact area at higher $T_{\mathrm{b}}$. We obtained values ranging from $k_{\mathrm{SiCu}} \simeq 2500 \mathrm{~W} \mathrm{~m}^{-2} \mathrm{~K}^{-1}(E=8 \mathrm{keV})$ to $\sim 3500 \mathrm{~W} \mathrm{~m}^{-2} \mathrm{~K}^{-1}(E=3.5 \mathrm{keV})$, as in previous analyses (Zhang et al., 2003, 2013).

The experimental vertical beam size is proportional to crystal slope error $\theta$, in good approximation. It has been measured via an imaging camera, versus time and $T_{\mathrm{b}}$, as shown in Fig. 9. It increases for about $10 \mathrm{~s}$, to reach its maximum value, and then decreases to its final equilibrium value exponentially, when the crystal temperature reaches $T_{\mathrm{b}}$. The same trend is predicted by equation (21), which has been used to fit the data.

To understand the dynamic behaviour, a Python code was written, based on the heat equation. The I16 crystal monochromator temperature was calculated iteratively, showing that $T_{\mathrm{p}}$ reaches its maximum value in fractions of a second, with $T_{\mathrm{b}}$ static. Successively, both $T_{\mathrm{p}}$ and $T_{\mathrm{b}}$ increase at a similar rate, to reach a steady state, with time scales of about $1 \mathrm{~min}$. Fig. 9

Figure 9 data [equation (21)].

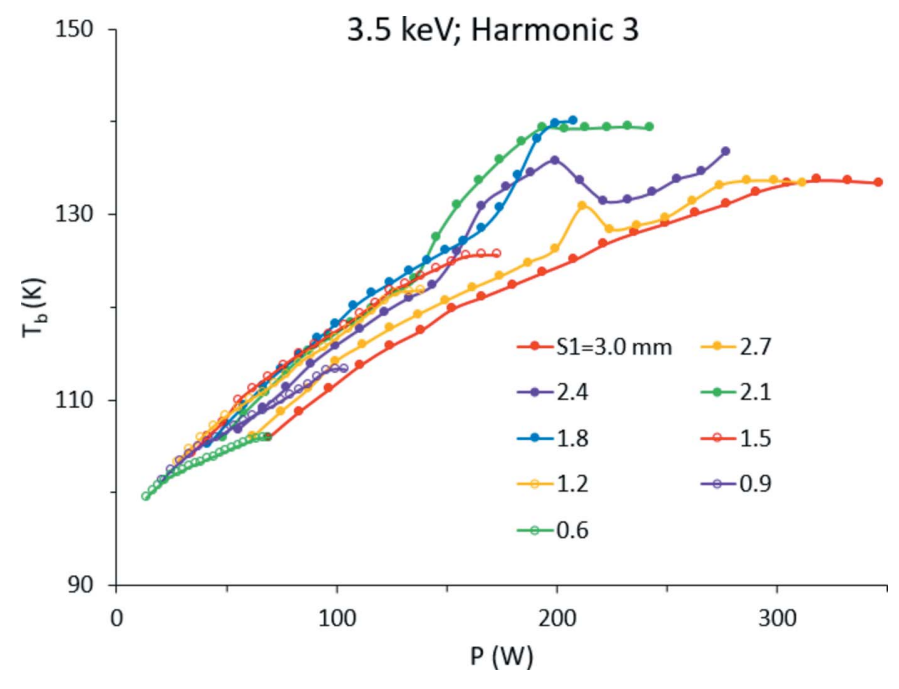

Figure 8

Steady state values of $T_{\mathrm{b}}$ versus calculated power for $E=3.5 \mathrm{keV}$ and $5.0 \mathrm{keV}$ and different slit size.

shows that the beam size changes continuously until it reaches the equilibrium state. This confirms that both $T_{\mathrm{p}}$ and $T_{\mathrm{b}}$ contribute to the surface deformation.

Equilibrium values of vertical beam size versus absorbed power are shown in Fig. 10 (top). Local minima of the beam size are of order $20-30 \mu \mathrm{m}$, which is twice the value expected from modelling the monochromator as a flat perfect crystal. Different regimes are observed: a smooth increase is measured at low power; beam size then decreases at medium power values, reaching its minimum at the 'sweet spot'; a steep increase to $200-400 \mu \mathrm{m}$ with increasing the heat load. This is a critical scenario, presenting a beam-size linear trend of around an order of magnitude larger than at low power. In summary, crystal deformation does not simply increase linearly with absorbed power. Similar trends for the slope error are reported (Zhang et al., 2003, 2013; Huang et al., 2014; Brumund et al., 2021; Huang \& Bilderback, 2012), as this is a universal behaviour. In this study, critical values for the 'sweet spot' are in the range $P \simeq 80$ to $180 \mathrm{~W}$ depending on values of
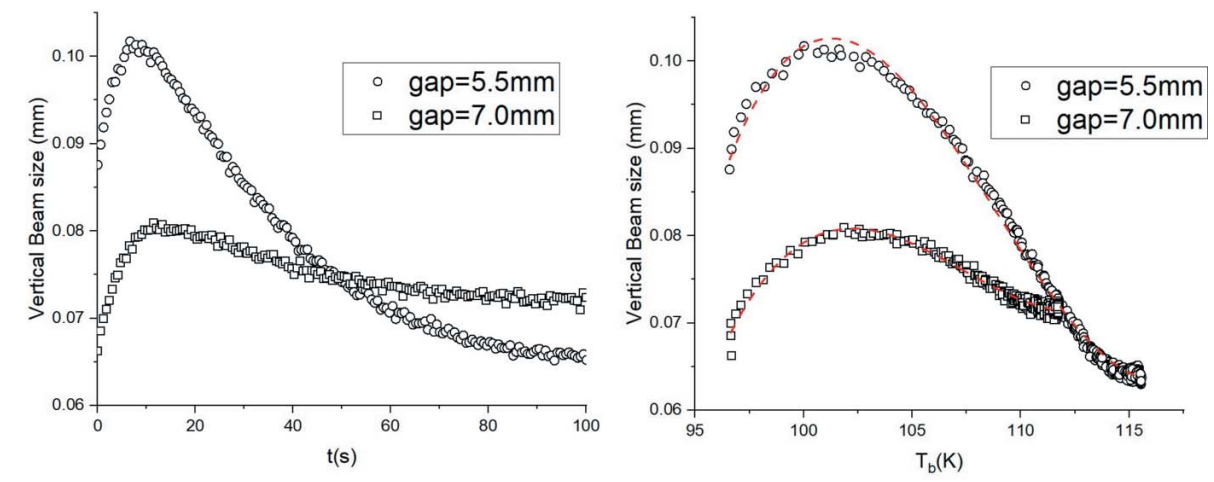

Vertical beam size versus time (left) and measured crystal temperature (right) for two gap values at $E=8.0 \mathrm{keV}$. The red dashed lines in the right-hand plot show the third-order polynomial fit of the 

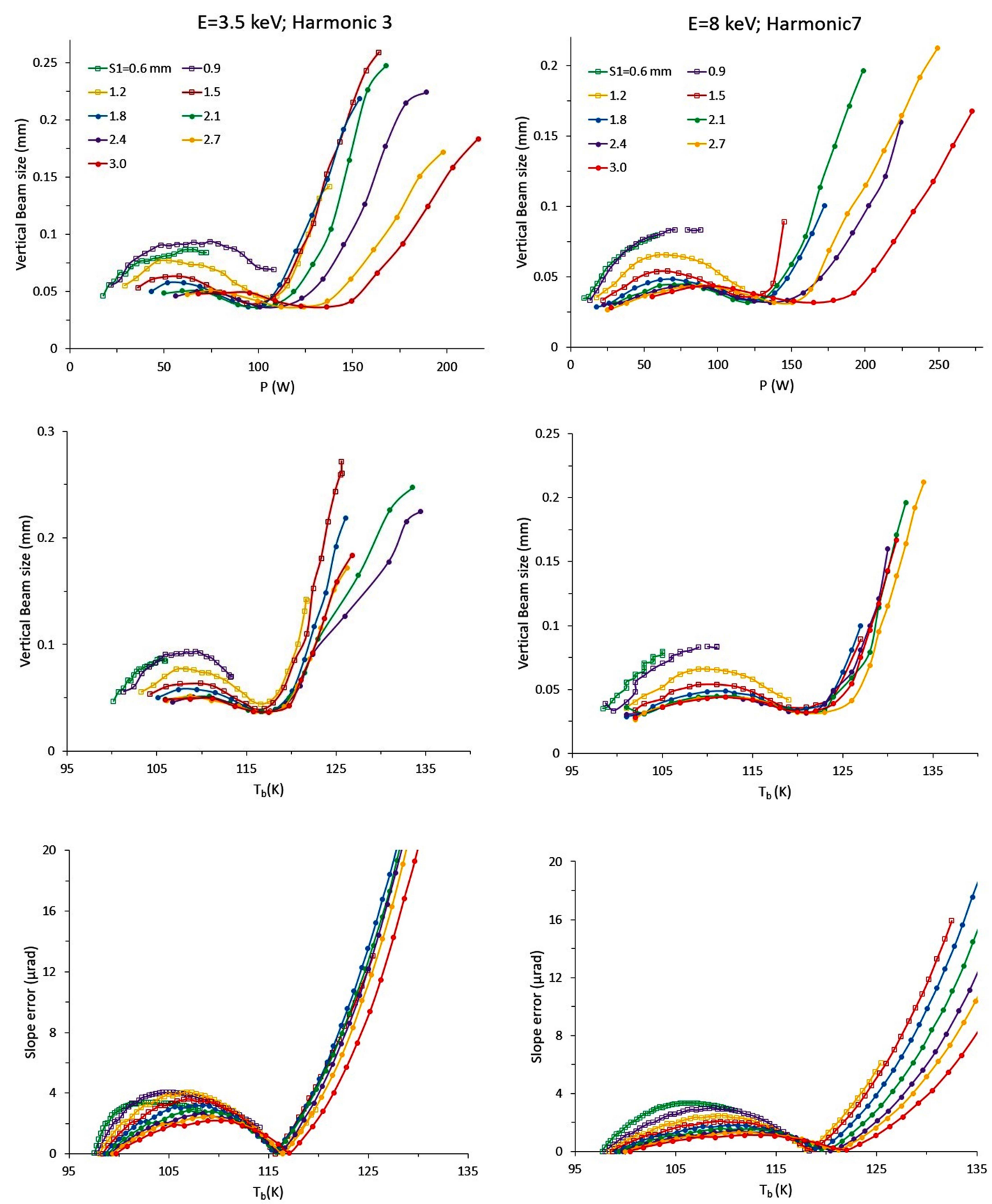

Figure 10

Measured vertical beam size at the focal position versus calculated power (top) and $T_{\mathrm{b}}$ (middle) for typical power conditions. Bottom: slope error from the model in Section 2 at $E=3.5 \mathrm{keV}$ (left) and $8 \mathrm{keV}$ (right). Legends indicate the vertical opening of $\mathrm{S}_{1}$.

$P_{\mathrm{d}}$ and of incident beam size, whilst they are of order $300 \mathrm{~W}$ to $400 \mathrm{~W}$ in Zhang et al. (2013). The large difference is due to the very different cooling geometries.

A better parameter to control crystal deformation is the $T_{\mathrm{b}}$ temperature. The vertical beam size data in Fig. 10 (centre) indicate that the scenario of minimum deformation is achieved in the range $T_{\mathrm{b}}=116 \mathrm{~K}\left(P_{\mathrm{d}}=11 \mathrm{~W} \mathrm{~mm}^{-2}\right)$ to $121 \mathrm{~K}\left(P_{\mathrm{d}}=\right.$ $6 \mathrm{~W} \mathrm{~mm}^{-2}$ ). The slope error from the model (Fig. 10, bottom) is a good fit to experimental data. A cooling design with accurate feedback is suggested to minimize the deformation and prevent any transition to the very high deformation regime.

\section{Conclusions}

On-going upgrades of synchrotron sources and instrumentation deliver brighter and brighter beams of X-rays for applications ranging from biology to chemistry, from physics to engineering. Large investments in new sources are accompanied by progress in optics, detector technology and data storage. Study of matter at the nano-scale with X-rays, which was unthinkable when the first third-generation sources were built, is now reality. Stability and perfection of crystal monochromators is paramount to conserve the flux and brightness of synchrotron beams. This model of temperature and crystal 
deformation in indirectly cryo-cooled $\mathrm{Si}$ crystals illustrates and explains the dependence of crystal thermal slope errors observed in several synchrotron monochromator studies. Used in conjunction with FEA, it has been used to finalize crystal design and cooling at Diamond Light Source and to assess the resilience of these new optics for the future synchrotron machine.

\section{APPENDIX $A$}

\section{A1. Derivation of equation (3)}

Equations (1) and (2) are combined to obtain a secondorder differential equation for $T$,

$$
\frac{\partial^{2} T(t)}{\partial^{2} t}+\Gamma \frac{\partial T(t)}{\partial t}+\Lambda T(t)=\Omega
$$

where

$$
\begin{aligned}
& \Gamma=\frac{\kappa_{\mathrm{Si}}}{\mu_{\mathrm{Si}}}+\frac{\kappa_{\mathrm{Si}}+\kappa_{\mathrm{Cu}}}{\mu_{\mathrm{Cu}}}, \quad \Lambda=\frac{\kappa_{\mathrm{Si}} \kappa_{\mathrm{Cu}}}{\mu_{\mathrm{Si}} \mu_{\mathrm{Cu}}}, \\
& \Omega=\frac{\kappa_{\mathrm{Si}} \kappa_{\mathrm{Cu}}}{\mu_{\mathrm{Si}} \mu_{\mathrm{Cu}}} T_{\mathrm{LN} 2}+\frac{\kappa_{\mathrm{Si}}+\kappa_{\mathrm{Cu}}}{\mu_{\mathrm{Si}} \mu_{\mathrm{Cu}}} P, \\
& \kappa_{\mathrm{Si}}=k_{\mathrm{SiCu}} A_{\mathrm{SiCu}}, \quad \kappa_{\mathrm{Cu}}=k_{\mathrm{CuLN} 2} A_{\mathrm{CuLN} 2}, \\
& \mu_{\mathrm{Si}}=m_{\mathrm{Si}} C_{\mathrm{Si}}, \quad \mu_{\mathrm{Cu}}=m_{\mathrm{Cu}} C_{\mathrm{Cu}} .
\end{aligned}
$$

All other parameters are assumed to be independent of temperature and averaged over time and space. In practical cases, $k_{\mathrm{SiCu}}$ includes a contribution from the heat conductance of the indium foil. $T_{\mathrm{Cu}}(t)$ is assumed constant along the $\mathrm{Cu}-\mathrm{Si}$ and $\mathrm{Cu}-\mathrm{LN} 2$ contact surfaces.

Inserting equation (3) into equation (8),

$$
\begin{gathered}
T_{\mathrm{b}}=\frac{\Omega}{\Lambda}=T_{\mathrm{LN} 2}+\left(\frac{1}{\kappa_{\mathrm{Si}}}+\frac{1}{\kappa_{\mathrm{Cu}}}\right) P, \\
\frac{1}{\tau_{ \pm}}=\frac{\Gamma \pm\left(\Gamma^{2}-4 \Lambda\right)^{1 / 2}}{2} .
\end{gathered}
$$

Using equations (8), (9) and (10),

$$
\frac{1}{\tau_{+}}+\frac{1}{\tau_{-}}=\frac{\kappa_{\mathrm{Si}}}{\mu_{\mathrm{Si}}}+\frac{\mu_{\mathrm{Si}}}{\tau_{+} \tau_{-}} \frac{T_{\mathrm{b}}-T_{\mathrm{LN} 2}}{P} .
$$

\section{A2. Derivation of characteristic temperatures}

At low power, with $T(r)$ slowly varying, $k_{\mathrm{Si}}$ can be assumed constant and the solution to equation (4) is

$$
T(r)=T(0)-\frac{1}{k_{\mathrm{Si}}} \xi(r) \frac{P}{a},
$$

where

$$
\xi(r)=\frac{r}{8 a}, \quad r<a ; \quad \xi(r)=\left(\frac{1}{8}-\frac{r-a}{2 \pi r}\right), \quad r>a .
$$

The solution is: $T_{\mathrm{p}}=T_{\mathrm{b}}+P /\left(14.3 a k_{\mathrm{Si}}\right)$, where $T_{\mathrm{p}}=T(0)$ is the peak temperature.

At high incident power,

$$
\begin{aligned}
& T_{\mathrm{p}}=\frac{\left(\eta_{\mathrm{a}}-1\right) \Lambda_{1} \Lambda_{2}-\left(\Lambda_{1}+\eta_{\mathrm{a}} \Lambda_{2}\right) T_{\mathrm{a}}}{\left(\eta_{\mathrm{a}}-1\right) T_{\mathrm{a}}-\left(\eta_{\mathrm{a}} \Lambda_{1}+\Lambda_{2}\right)}, \\
& T_{\mathrm{a}}=\frac{\left(\eta_{\infty}-1\right) \Lambda_{1} \Lambda_{2}-\left(\Lambda_{1}+\eta_{\infty} \Lambda_{2}\right) T_{\mathrm{b}}}{\left(\eta_{\infty}-1\right) T_{\mathrm{b}}-\left(\eta_{\infty} \Lambda_{1}+\Lambda_{2}\right)},
\end{aligned}
$$

where $T_{\mathrm{a}}=T(r=a), \Lambda_{1}=34.4 \mathrm{~K}, \Lambda_{2}=158.4 \mathrm{~K}, \eta_{\mathrm{a}}=$ $\exp [\gamma(P / a)] ; \eta_{\infty}=\exp [\varepsilon(P / a)]$, where $\gamma=1.6 \times 10^{-6} \mathrm{~m} \mathrm{~W}^{-1}$, $\varepsilon=2.1 \times 10^{-6} \mathrm{~m} \mathrm{~W}^{-1}$.

Typical average values are $P=200 \mathrm{~W}, P_{\mathrm{d}}=10 \mathrm{~W} \mathrm{~mm}^{-2}$ and $a \simeq 2.5 \mathrm{~mm}$ at DLS. Equations $(6 a)$ and $(6 b)$ can be approximated as

$$
\begin{aligned}
& T_{\mathrm{p}}=T_{\mathrm{a}}+\gamma \frac{P}{a} \frac{\left(T_{\mathrm{a}}-\Lambda_{1}\right)\left(T_{\mathrm{a}}+\Lambda_{2}\right)}{\Lambda_{1}+\Lambda_{2}}, \\
& T_{\mathrm{a}}=T_{\mathrm{b}}+\varepsilon \frac{P}{a} \frac{\left(T_{\mathrm{b}}-\Lambda_{1}\right) \times\left(T_{\mathrm{b}}+\Lambda_{2}\right)}{\Lambda_{1}+\Lambda_{2}} .
\end{aligned}
$$

Integration of equations (14) over the beam footprint and the remaining Si bulk,

$$
\begin{gathered}
\int_{0}^{r^{\prime}<a} \frac{P_{\text {radial }}\left(r^{\prime}\right)}{A\left(r^{\prime}\right)} \mathrm{d} r^{\prime}=-\int_{T_{\mathrm{p}}}^{T(r)} k_{\mathrm{Si}}[T(r)] \mathrm{d} T(r), \quad r<a, \\
P \int_{0}^{r^{\prime}>a} \frac{1}{A\left(r^{\prime}\right)} \mathrm{d} r^{\prime}=-\int_{T_{\mathrm{a}}}^{T(r)} k_{\mathrm{Si}}[T(r)] \mathrm{d} T(r), \quad r>a,
\end{gathered}
$$

where $P_{\text {radial }}(r)=\left(\pi r^{2} / 4 a^{2}\right) P$. All parameters are in standard SI units. For a circular footprint, $A(r)=2 \pi r^{2}$; numerical integration is performed in the case of an elliptical footprint,

$$
A\left(r_{1}, r_{2}\right)=2 \pi\left[\frac{r_{2}^{3.2}+2 \times\left(r_{1} r_{2}\right)^{1.6}}{3}\right]^{1 / 1.6} \text { for } r_{2}<r_{1} \text {. }
$$

Here, $r_{1}$ and $r_{2}$ are the semi-major and semi-minor axes of the elliptical beam footprint. $k_{\mathrm{Si}}$ is assumed to be constant at low power, when temperature changes only slightly inside the crystal. However, at high power, one of the following functions can be used,

$$
\begin{gathered}
k_{\mathrm{Si}}(T) \simeq 8.3 \times 10^{6} T^{-2}, \\
k_{\mathrm{Si}}(T)=\frac{1490}{(T / 100+0.62)^{2}-0.93},
\end{gathered}
$$

with $T$ expressed in $\mathrm{K}$, and $k_{\mathrm{Si}}(T)$ in $\mathrm{W} \mathrm{m}^{-2} \mathrm{~K}^{-1}$. These functions have been obtained from fitting the experimental data of Touloukian et al. (1971) in the range of interest $80 \mathrm{~K}$ to $180 \mathrm{~K}$. Both forms can be integrated analytically; however, equation (15) leads to simpler expressions,

$$
\frac{1}{T(r)}-\frac{1}{T(0)}=1.5 \times 10^{-8} \sigma(r) \frac{P}{a}
$$

$$
\sigma(r)=\frac{r}{a}, \quad r<a ; \quad \sigma(r)=\left(1+\frac{4}{3} \frac{r-a}{r}\right), \quad r>a,
$$

$$
\frac{T_{\mathrm{p}}-T_{\mathrm{b}}}{T_{\mathrm{p}} T_{\mathrm{b}}}=3.5 \times 10^{-8} \frac{P}{a} .
$$


Use of the more accurate expression in equation (16) leads to

$$
T(r)=\frac{\left[\rho(r) \Lambda_{1}+\Lambda_{2}\right] T_{0}+[\rho(r)-1] \Lambda_{1} \Lambda_{2}}{[\rho(r)-1] T_{0}+\left[\rho(r) \Lambda_{2}+\Lambda_{1}\right]},
$$

where

$$
\begin{aligned}
& \rho(r)=\exp \left(\gamma P \frac{r}{a^{2}}\right), \quad T_{0}=T_{\mathrm{p}}, \quad r \leq a, \\
& \rho(r)=\exp \left(\varepsilon P \frac{r-a}{a r}\right), \quad T_{0}=T_{\mathrm{a}}, \quad r>a .
\end{aligned}
$$

Therefore, $T_{\mathrm{p}}$ and $T_{\mathrm{b}}$ can be calculated for any cooling design.

\section{A3. Derivation of thermal strain}

The temperature dependence of the Si thermal expansion coefficient (Middelmann et al., 2015) in the range 80-240 K leads to the following expression,

$$
\Delta L(T)=A T^{4}+B T^{3}+C T^{2}+D T+E,
$$

with parameters obtained from data fitting: $A=-1.5108 \times$ $10^{-13} \mathrm{~m} \mathrm{~K}^{-4} ; B=9.5288 \times 10^{-11} \mathrm{~m} \mathrm{~K}^{-3} ; C=-1.2778 \times$ $10^{-8} \mathrm{~m} \mathrm{~K}^{-2} ; D=-1.4974 \times 10^{-7} \mathrm{~m} \mathrm{~K}^{-1} ; E=-1.7656 \times$ $10^{-4} \mathrm{~m}$.

At low power,

$$
\begin{aligned}
\Delta L_{\mathrm{p}, \mathrm{b}}= & (4 A p) T_{\mathrm{b}}^{3}+\left(6 A p^{2}+3 B p\right) T_{\mathrm{b}}^{2} \\
& +\left(4 A p^{3}+3 B p^{2}+2 C p\right) T_{\mathrm{b}} \\
& +\left(A p^{4}+B p^{3}+C p^{2}+D p\right)
\end{aligned}
$$

where $p=P /\left(14.3\right.$ a $\left.k_{\mathrm{Si}}\right)$.

The $T_{\mathrm{b}}^{3}$ polynomial term in the slope error is observed experimentally.

\section{Acknowledgements}

We acknowledge Diamond Light Source for time on beamline I16. The authors are grateful to Pablo Sánchez-Navarro for many discussions on FEA.

\section{References}

Antimonov, M. A., Khounsary, A. M., Sandy, A. R., Narayanan, S. \& Navrotski, G. (2016). Nucl. Instrum. Methods Phys. Res. A, 820, 164-171.

Brumund, P., Reyes-Herrera, J., Detlefs, C., Morawe, C., Sanchez del Rio, M. \& Chumakov, A. I. (2021). J. Synchrotron Rad. 28, 91-103.

Cheng, X., Zhang, L., Morawe, C. \& Sanchez del Rio, M. (2015). J. Synchrotron Rad. 22, 317-327.

Chumakov, A., Rüffer, R., Leupold, O., Celse, J.-P., Martel, K., Rossat, M. \& Lee, W.-K. (2004). J. Synchrotron Rad. 11, 132-141.

Collins, S. P., Bombardi, A., Marshall, A. R., Williams, J. H., Barlow, G., Day, A. G., Pearson, M. R., Woolliscroft, R. J., Walton, R. D., Beutier, G., Nisbet, G., Garrett, R., Gentle, I., Nugent, K. \& Wilkins, S. (2010). AIP Conf. Proc. 1234, 303-306.

Dolbnya, I. P., Sawhney, K. J. S., Scott, S. M., Dent, A. J., Cibin, G., Preece, G. M., Pedersen, U. K., Kelly, J. \& Murray, P. (2019). J. Synchrotron Rad. 26, 253-262.

Huang, R. \& Bilderback, D. H. (2012). Proc. SPIE, 8502, 85020B.

Huang, R., Bilderback, D. H. \& Finkelstein, K. (2014). J. Synchrotron Rad. 21, 366-375.

Kazimirov, A., Revesz, P. \& Huang, R. (2007). Nucl. Instrum. Methods Phys. Res. A, 576, 422.

Kazimirov, A., Revesz, P. \& Huang, R. (2008). Proc. SPIE, 7077, 707702.

Marot, G., Rossat, M., Freund, A., Joksch, S., Kawata, H., Zhang, L., Ziegler, E., Berman, L., Chapman, D., Hastings, J. B. \& Iarocci, M. (1992). Rev. Sci. Instrum. 63, 477-480.

Middelmann, T., Walkov, A., Bartl, G. \& Schödel, R. (2015). Phys. Rev. B, 92, 174113.

Revesz, P., Kazimirov, A. \& Bazarov, I. (2007). Nucl. Instrum. Methods Phys. Res. A, 582, 142-145.

Rutishauser, S., Rack, A., Weitkamp, T., Kayser, Y., David, C. \& Macrander, A. T. (2013). J. Synchrotron Rad. 20, 300-305.

Tanaka, T. \& Kitamura, H. (2001). J. Synchrotron Rad. 8, 1221-1228.

Touloukian, Y., Powell, R., Ho, C. \& Klemens, P. (1971). Thermophysical Properties of Matter, Vol. 1, Thermal Conductivity Metallic Elements and Alloys.

Yates, B., Hu, Y. \& Nagarkal, V. (2010). Proc. SPIE, 7802, 78020U.

Yovanovich, M. M. (2005). IEEE Trans. C. Packag. Technol. 28, 182206.

Zhang, L., Lee, W.-K., Wulff, M. \& Eybert, L. (2003). J. Synchrotron Rad. 10, 313-319.

Zhang, L., Sánchez del Río, M., Monaco, G., Detlefs, C., Roth, T., Chumakov, A. I. \& Glatzel, P. (2013). J. Synchrotron Rad. 20, $567-$ 580 . 